\title{
"Woodlands" - a Virtual Reality Serious Game Supporting Learning of Practical Road Safety Skills.
}

Krzysztof Szczurowski

TU Dublin Blanchardstown

Matt Smith

TU Dublin Blanchardstown

Follow this and additional works at: https://arrow.tudublin.ie/grapc

Part of the Computer Engineering Commons, and the Graphics and Human Computer Interfaces Commons

\section{Recommended Citation}

Szczurowski, K. \& Smith, M. (2018) "Woodlands" - a Virtual Reality Serious Game Supporting Learning of Practical Road Safety Skills, 2018 IEEE Games, Entertainment, Media Conference (GEM). August 15-17, 2018, Galway, Ireland.

This Article is brought to you for free and open access by the Graphics and Gaming Research Group at ARROW@TU Dublin. It has been accepted for inclusion in Conference Papers by an authorized administrator of ARROW@TU Dublin. For more information, please contact arrow.admin@tudublin.ie, aisling.coyne@tudublin.ie, gerard.connolly@tudublin.ie.

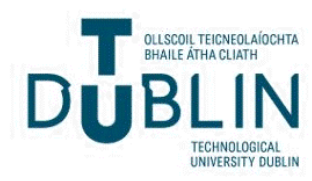




\title{
"Woodlands" - a virtual reality serious game supporting learning of practical road safety skills.
}

\author{
Krzysztof Szczurowski, Matt Smith \\ Department of Informatics \\ Institute of Technology Blanchardstown ${ }^{1}$ \\ Dublin, Ireland \\ krzysztof.szczurowski@student.itb.ie,matt.smith@itb.ie
}

\begin{abstract}
In developed societies road safety skills are taught early and often practiced under the supervision of a parent, providing children with a combination of theoretical and practical knowledge. At some point children will attempt to cross a road unsupervised, at that point in time their safety depends on the effectiveness of their road safety education.
\end{abstract}

To date, various attempts to supplement road safety education with technology were made. Most common approach focus on addressing declarative knowledge, by delivering road safety theory in an engaging fashion. Apart from expanding on text based resources to include instructional videos and animations, some stakeholders (e.g.: Irish Road Safety Authority) attempt to take advantage of game-based learning [1]. However, despite the high capacity for interaction being common in Virtual Environments, available game-based solutions to road safety education are currently limited to delivering and assessing declarative knowledge.

With recent advancements in the field of Virtual Reality (VR) Head Mounted Displays, procedural knowledge might also be addressed in Virtual Environments. This paper describes the design and development process of a computer-supported learning system that attempts to address psycho-motor skills involved in crossing a road safely, changing learners' attitude towards road safety best practices, and enabling independent practice of transferable skills.

By implementing game-based learning principles and following best practice for serious game design (such as making educational components essential to successful game-play, or instructional scaffolding) we hope to make it not only more effective, but also engaging, allowing us to rely on learners' intrinsic motivation [2], to increase their independent practice time and provide them with feedback that will help to condition safe behaviour and increase retention.

Presence in Virtual Reality might evoke responses to Virtual Environment as if it was real (RAIR) [3] and enable learners to truly experience learning scenarios. In consequence leading to formation of autobiographical memories constructed from multisensory input, which should result in an increased knowledge retention and transfer [4].

Keywords-Virtual Reality; VR; Road Safety; Serious Game; Experiential Learning; Game-Based Learning; Virtual Environment;

\section{INTRODUCTION}

Arguments on behalf of experiential learning can be traced back to Confucius and Aristotle [5], and the refined model published by Kolb emphasised observation and reflection upon the experience as a source of abstract knowledge [6]. As much as there might be some debate around the value of the knowledge acquired through experience for abstract concepts, its role in developing procedural knowledge (particularly in case of skills involving psycho-motor activity or developing reflexes) is unquestionable. Conceptually there seems to be a strong division between training and learning, the latter is often perceived as the acquisition of new information as compared to reinforcement of practical skills that can be addressed through training. However, it is easy to identify a number of cases where the combination of the two might be required to achieve the best results. In such cases the proportion of time dedicated to declarative versus procedural knowledge will often depend on the time that is available to perform the activity and the amount of time available for decision making. Complex tasks that require strategizing and planning will naturally benefit from stronger emphasis on theoretical component and declarative knowledge, while tasks relying on reflexes will benefit from increased amount of behavioural conditioning addressed through rehearsal and practice [7]. Pedestrian road safety education can certainly benefit from addressing declarative knowledge, but just as it is the case of sports an increased performance will likely be achieved if a strong emphasis is placed on practical components. Crossing a road is an activity that often happens without a lot of attention dedicated to it, presumably this is even more true in the

${ }^{1}$ This paper describe the work submitted as a Final Year Project for the award of Bachelor of Arts (Hons) in Creative Digital Media to the Institute of Technology Blanchardstown (ITB). Writing of this publication was partially supported by the ITB Postgraduate Seed Fund. 
case of children. Therefore, it is important to develop habits that will invoke safe behaviour in children crossing a road.

Practical approach to road safety training has a number of issues that can prevent such instruction from taking place in a physical environment. The risk involved in crossing a road, even in scenarios where traffic conditions can be controlled (e.g.: private road and drivers designated for that purpose) cannot guarantee safety of the participants.

Virtual learning environments have an advantage over physical environments by providing a safe space for practicing skills that would otherwise impose a risk of a serious (possibly fatal) injury on the learner [8]. Although not all virtual environments equally capable of inducing presence and consequent real life responses to virtual stimulus [9]. Research suggest that Head Mounted Displays (HMD) have an unprecedented capacity for inducing presence [10]. This is likely due to a fact that competing stimuli flowing from a physical environment is vastly reduced [11] (compared to flat screens) as the visual and (often) auditory streams are fully occupied by the stimulus flowing from the artificial environment.

An added benefit of using Head Mounted Displays is an availability of the positional tracking of a users' head that is needed for accurate translation of the displayed content in reference to users' head movement. In the context of road safety education and crossing a road this feature of the system can prove itself useful for evaluating learners' performance, and might also be used to condition behaviour by enforcing desired head movement before progression through the learning scenario is allowed.

The large number of attempts available to a learner, combined with constant feedback, offers means for developing correct habits through repetition of desired behaviours and reinforcement through a reward system that can increase learners' intrinsic motivation to perform to the best of their abilities [12].

This manuscript describes one potential approach to implementing game-based learning into road safety education. First a brief review of game-based learning methodologies and concepts applicable to the development of a computer supported learning system designed to teach and practice road safety skills to children is provided. Potential limitations of currently available hardware that can be used for such purpose is discussed, together with hypothetical application of emerging technologies (such as eye-tracking or omni-directional treadmills) that could be used to improve results in the future is also presented.

\section{BACKGROUND AND LITERATURE REVIEW}

There's a body of research attempting to explain how and why should we use games in education. Some researchers like Marc Prensky [13] or Richard Van Eck [14] argue that the 'net generation' is fundamentally different to their parents. Educators can no longer take students for granted, and have to become competitors on the market for our attention. Contemporary learners who changed books and illustrations for interactive games seems to be less capable of learning for the sake of learning and expect knowledge to be applicable to make it worth their effort. One early publication challenging academics in what is now the field of 'serious games' was Malone's article, the title of which asked "What makes computer games fun?" [15]. Malone studied how the design elements making games captivating can be used to make learning with computers more engaging. Around the same time a range of research and development was under way by people like Burton \& Brown [16] into educational games such as 'How the West Was Won', a.k.a. 'West'.

Much of the educational theories and techniques built around Vygotskian social constructivism assume that (a) young children are the learners and (b) a human teacher is observing, interacting with, engaging in dialogue with the learners. Serious games may be targeted at older children or adult learners, and in many cases serious game may be designed for such learners to work alone. This raises the question (challenge!) of how to embed the positive benefits of scaffolding and social constructivism in elearning systems without an active, present, human educator. One direction e-learning researchers have followed is that of 'intelligent tutors'. Computer programs can do much more than simply facilitate game play by learners. As previously described, the West system also offered a form of coaching. The computer's modelling of student beliefs (and perhaps misconceptions) allows for a wide range of rich interactions and interventions between computer system and the learner. Such 'intelligent tutors' can be explicit in their interactions, or the intervention may not be noticed by the student. Examples of explicit learning interventions by intelligent tutors can take many forms, e.g.: recommendations of how pairs of students might work together on a range of topics, based on analyses of their respective strengths and weaknesses in their learner models [17]. A more detailed review of both games and learning in general, and computer-based serious games can be found in "Serious Games and Edutainment Applications" [18].

\section{MOTIVATION AND AIMS OF THE SERIOUS GAME}

During the informal functionality tests of the "Crosswalk 2" simulator [19], a number of participants demonstrated behaviors that can be classified as involuntary reflexes. When collision with oncoming virtual traffic was inevitable, some participants braced for impact by reaching out with their hands to where the car bonnet would be. Based on this observation we hypothesize these involuntary reflexes, are caused by participants' high level of presence in that Virtual Environment. There's a body of research and empirical evidence, demonstrating that Virtual Reality systems might have the capacity to trick the human brain into believing (at least on a subconscious level) that the Virtual Environment is their physical, non-mediated environment, invoking life like behaviors and reflexes [20]. Our understanding of presence in mediated environments is still largely incomplete and based primarily on qualitative analysis. Nevertheless, judging by how successful Virtual Reality exposure therapies for phobic behaviors can be (on a par with in vivo therapy) [9], we believe this phenomenon to be true, however further research into presence, particularly into quantitative methods for measuring presence is necessary to further our understanding of the potential Virtual Reality might have in training and education [21]. In our view the high 
potential for inducing presence in Virtual Reality applications can be beneficial to developing procedural knowledge.

As famous research in neurology on patient H.M. [22] suggests, procedural and declarative knowledge are not as connected as some might believe. Severe damage to hippocampus impairing patients' capacity for forming new memories and developing any new declarative knowledge, doesn't seems to affect their capacity for developing new procedural knowledge as it is largely controlled by Basal Ganglia [23]. For example, despite the fact that H.M. didn't remember practicing mirror drawing during previous trials, his capacity for psycho-motoric learning was similar to participants with no neurological disorders.

These findings are reflecting what can be observed in sports: expert level of declarative knowledge, doesn't necessarily translate directly into procedural knowledge. Regardless of how well one might know the methodology for performing a perfect golf swing, or even underlying biomechanics and all the physics laws involved, a significant amount of practice to achieve psycho-motoric mastery of the skills involved is needed. Indepth knowledge of basketball rules can make you a great referee but not necessarily a world-class basketball player. Although, as some research on performance during tactical decision making in games involving a ball suggests that explicit learning tends to outperform implicit learning in highly-complex scenarios, while the opposite is true for low-complexity situations [7]. Therefore, we hypothesize that both procedural and declarative learning are necessary to address safe road crossing skills that tend to vary in complexity but rarely involves complex tactics.

We argue that humans rely primarily on procedural knowledge when crossing a road, after all most of us don't have to remind ourselves about looking both ways before making the decision to step onto a road, and children are probably even less likely to refer to a step by step instruction that they have learn in school. As such children are in a higher risk group of being involved in a traffic accident while crossing a road unsupervised due to their lack of experience in performing the task, and possibly other factors (e.g.: prefrontal cortex being still in the developmental stage preventing them from correctly connecting their actions with consequences [24]). Therefore, developing safe habits that can be executed early in a children pedestrian 'career' is of great importance, despite the fact that the number of children being involved in pedestrian accidents is relatively low.

A potential solution to this problem can be delivered through the hardware available today, as it is now possible to address psycho-motoric skills and train all the systems that are involved in safe road crossing in a computer simulated environment, as the perceptual and cognitive processes might also benefit from practice in Virtual Reality. Although the hardware in itself is not going to produce such results, a careful consideration has to be given to the design of the learning support system to not only accommodate training but also be engaging for the student.

In sports, psycho-motoric skills are developed through prolonged practice and repetition. Athletes dedicate thousands of hours in order to achieve psycho-motoric mastery of the skillset needed in their discipline [25]. Crossing a road is not a very exciting or rewarding experience, and some could even describe it as mundane. Therefore, it seems that crossing a road might not have the same appeal as playing rugby or swimming. Therefore, a virtual reality game based approach seems like the right path for increasing participation and engagement in training scenarios [8].

Apart from increasing student engagement game-based learning can also include elements of a problem based learning. In the latest iteration of the serious game for teaching road safety skills (further referred to as "Woodlands") every crossing is a problem to solve, and the learner has to figure out the solution to each problem by themselves. To facilitate instrumental conditioning looking right, left and right again is enforced on players, however players will quickly learn that sometimes this strategy might not be sufficient to cross the road, at times player might have to repeat this process until there's no traffic in their proximity. Looking right, left and right again is not a part of the safe cross code taught in Ireland. Instead it's being subsidized with "don't hurry, stop and wait" followed by "look all around and listen". A possible explanation to this is the fact that simply looking right, left and right again in certain scenarios might not be sufficient. Finding the right solution to a puzzle in a problem based scenario, allow learners to associate meaning to theory facilitating deeper level of understanding consequently leading to higher level of performance and retention.

\section{DESIGN OF THE SERIOUS GAME}

During the design phase following educational goals have been identified:

1) Practical components: car spotting, checking both sides for incoming traffic, identifying safest place to cross a road.

2) Theoretical components: road safety code [26], importance of wearing high visibility safety clothing, and using traffic lights controlled crossings.

Given that children's lives are on the line a number of potential issues had to be carefully considered in the context of how the game design might affect children perception of the road traffic and safety. Therefore, the first principle for designing "Woodlands" was to avoid any possibilities for promoting unsafe behaviour or giving a player an option to 'chance it'. Therefore, despite the fact that 'AI' of cars in Woodlands is perfectly capable of detecting the player in front of them and trigger breaking or evasive procedure, cars in Woodlands do not stop, or even slow down, when the player walks onto a road, without previously activating assistive components such as the lollipop lady, traffic lights or a high visibility jacket. This design decision was made to avoid a possibly that learners could develop an assumption that traffic will yield for them unconditionally, every time they step onto a road, which could lead to a tragedy in real life. Probably the biggest challenge in complying with the 'no reinforcement of undesired behaviour' principle was evident around the concept of 'lives'. Most games rely on multiple 'lives' and this is partially where the educational power of games originate, as the ability to reattempt a challenge with no, or minor penalty associated with poor performance, allow players to practice until their level of mastery becomes sufficient to succeed. Range of possible 
solutions were considered and evaluated against the principle of avoiding false or unsafe associations with road traffic. Multiple lives deducted on each 'death' until it reaches zero, when the game either restarts or takes the player back to the last checkpoint, was deemed unusable as it could violate the principle mentioned above. One live and restart or return to waypoint seemed more feasible. A time penalty preventing the player from accessing the game for a longer period of time (e.g.: 24hour) after collision with traffic provides not only a severe penalty for losing, emphasising consequences of wrong decision making at the road crossing, but also enable more control over practice schedule by distributing it over a longer time span, which can be beneficial to both performance and retention [27]. In addition, it would also reduce the time children spent playing the game in a single session, and since the consequences of prolonged VR exposure are still largely unknown, restricting the play time available in a single sitting is a safer approach to take. Another possible solution that was considered was to introduce punishment reinforcement by inducing nausea in a player during a collision with a car, by spinning virtual cameras around its own axis multiple times. However, due to possible ethical issues associated with purposeful induction of simulator sickness in underage participants, this approach was abounded, despite being probably most in line with required goals.

The core mechanism used to reinforce correct behaviors in "Woodlands" is operant conditioning. The player has to activate a hot-spot on their right side, next turn their head left to activate another hotspot and turn right again before they can step onto a road [see fig. 1]. Once this action is completed player is 'rewarded' through a praise delivered by a non-playable character who follows the player through the first level. As the player progress through the game, feedback frequency decrease over time. Non-playable character (NPC) act as an intelligent tutoring system providing the player with subtle clues when they can't figure out how to progress through the game on their own. "Woodlands" scaffolding system does not alter difficulty of the task but provides audio cues to guide the player instead. For example: if player spent too much time trying to step onto a road, the lollipop lady NPC [see fig $3 \& 4$ ] will instruct the player to "remember, always look right, left, and right again, before you cross a road".

There is more to safe road crossing than just a motor skill of looking both ways. It also requires perception, cognition and decision making.

\section{A. Perception}

Players perceptual system is being trained through:

- listening (the engine sound of the cars in "Woodlands" is mimicking real world vehicle sounds),

- $\quad$ and observation (cars are abstract but their geometric shapes are derived from simplified real world cars geometry [see fig. 2]).

In 'Woodlands' spotting cars is an activity that player have to engage in, to progress through certain points in the story line. Looking directly at the car results in their 'essence' being moved towards the NPC charging their special skill and also making them larger. In the future this component should be expanded to accommodate more "car spotting" game play. "Woodlands" doesn't use points therefore this could be addressed through an achievement system that would grant player with a trophy when, for example, they have spotted 100 blue cars.

\section{B. Cognition}

Cognitive system is addressed every time the player has to cross a road. Every next crossing is a slightly harder challenge, where player has to constantly evaluate incoming traffic in order to make a decision to cross the road, just like in real life, based on the input received from their perceptual system and their knowledge of road safety.

Difficulty progression can be addressed through modulation of parameters such as: speed of the traffic, magnitude of directions from which the traffic can come from, amount of obstacles obstructing the view, presence of assistive technologies (the 'lollipop lady' NPC, High Visible jacket, or traffic lights)

\section{Motor skills}

Motor system is exercised every time the player has to cross a road. Forcing a learner to look right, left and right again should support development of habits that will directly transfer to a real world behaviour.

To take advantage of mirror neurons and social-cognitive theory NPC's characters can themselves demonstrating correct behaviours to a player to aid the process of developing correct habits through imitation.

\section{Declarative knowledge}

The safe cross code from the Irish Road Safety Authority (RSA) is included in the game design to align its objectives with the Irish curriculum. To increase their appeal and learners interest in the content pieces of the code are scattered across all levels and presented to the player in a slightly sinister fashion: "keep watching", "look for a safe place", "look all around and listen", "don't hurry, stop and wait", "walking straight across", "you let all the traffic pass you". Fragmentation of the code together with a whispering narration aims to creates a sense of unease and mystery. Fragments of the code are presented as collectible gems that are stored on the bracelet that the player is wearing after being found in the environment. Player can access any piece of the code that they have found by looking directly at the gem item on their wrist. When player collects all the pieces, their goal is to solve the puzzle and put each gem with a corresponding piece of the code into the solution:

\section{" $1,2,3$, Safe Cross,}

4,5,6, Safe Cross,

1,2,3,4,5,6, Safe Cross Code

Remember, one, (...)

two, (...),

three, (...) before you cross the road,

remember, four, (...)

five, then (...)

six, (...), 


\section{that's the Safe Cross Code!!!!!”}

By delivering the theory component in such way, retention should be increased by making the player acquire and reconstruct knowledge themselves, taking advantage of cognitive constructivism principles [28].

Similarly, a high visible jacket that is available to the player at the final stages of the game is a power-up in game object, greatly assisting the player with safe crossing by slowing down incoming traffic. By turning high-vis jacket into a reward object it should be possible to increase positive attitude among children towards wearing one in real life.

\section{E. Empathy}

Virtual Reality provides a unique opportunity of experiencing life through the eyes of someone else. Some early work in the field demonstrate promising potential that VR has for inducing empathy [29]. By being placed on a futuristic wheelchair with clunky tank controls the player might become more aware of some of the everyday struggles, that a disabled person might face. Apart from increased empathy this design was introduced also to attempt to overcome the limitations of the input controller that is currently used (game pad). Integration of this feature into the story line, should make the player aware that they are on a wheelchair as a result of a car accident, giving children a small taste of how much more difficult their life might become if they do not pay attention while crossing a road. However, this component is still under evaluation and might be removed from the final design.

\section{F. Intrinsic motivation for exploration}

In order to make Woodlands more appealing and welcoming, elements of the game environment that were not essential to safe road crossing were abstracted and stylised, to create an extraordinary world, placed high above the clouds on the huge trees growing off the floating islands. Cars [see fig. 2] and traffic lights have been stylised to create a coherent wooden look and feel to the overall environment. During the Dice conference in 2010 Jesse Schell [30] made a convincing argument on behalf of players craving to return to nature, as a result of our detachment from it through a growing presence of technology in our lives. Shell attempts to explain the new trend of games entering a physical realm, forcing players to go outside and play in the real world, although the Alternate Reality Game (ARG) approach is not feasible in this content, there were still lessons to be learned here. Therefore, the first change to the original concept of housing blocks was an introduction of wooden textures applied to them, and in the final iteration housing blocks were replaced with huge habitable trees.

\section{G. Working around hardware limitations}

Initial hardware setup available during the development stage was falling short from minimum specification for pc based VR applications, therefore the load on the Graphic Processing Unit (GPU) had to be kept as low as possible. This limitation influenced a number of design decisions, for examples car models are kept at sub 200 vertices and use grey-scale low resolution textures that are later altered by changing albedo property of their material. Cars were particularly challenging as there is constantly quite a lot of them present in the environment, most of them are in motion, and rarely outside of player's field of view. In result they couldn't be statically batched or occluded. As a result, cars geometry still resembles real world cars, but they have no wheels or mirrors or anything else that is not essential to recognising the basic shape of a car.

\section{H. User Interface in VR for maximised presence}

User interface (UI) in first person perspective (FPP) video games is often utilising heads-up displays (HUD). However, we hypothesise that HUD UI could potentially be a distraction and result in reduced presence, which could have a negative impact on the effectiveness of training in virtual environment. As such, part of the UI has been moved onto the players' body. For example, each piece of the safety code ends up on players' wrist in a form of a bracelet. Interactions with the environment are gazed based and states are visible on the object the player is interacting with. For example, to turn on the traffic lights player has to look at the box (placed where the traffic lights button would normally be) and keep it in the centre of their field of view until it gradually turns from red to green. Gaze-based interactions are new to vast majority of players with no VR experience, therefore audio clues are provided to explain the concept to the player.

Although tests have shown that players need more guidance during navigation, originally pickup objects were supposed to do that job, but they were too few to effectively do that. Another pickup object should be introduced that will serve as a path indicator or a map based navigation system will have to be designed and implemented in the future.

\section{CORE COMPONENTS IMPLEMENTED IN THE GAME}

1) Crossing a road is only possible after player looks right left and right again (adjusted for UK/Ireland left side traffic).

2) Spotting cars has been implemented as one of core components necessary for successful gameplay. To add some appeal to it, performing this action will charge NPC's special power.

3) Quotes from the safe cross code have been turned into pickup objects.

4) Intelligent tutoring is provided by a non playable character following (and sometimes leading) the player through the game, at the time of writing this paper two NPC's were developed with a unique set of special abilities:

a) Lollipop lady (power to stop the traffic completely until the player reaches other side of the road, and the ability to shrink the player once charged with energy, player has to charge her by spotting cars)

b) Dog (Male character, currently inaccessible, special powers of slowing down time when the player is close to being hit by a car when charged - after each use player has to recharge this NPC by spotting cars or he will remain in the state of "agony") 
Environment has been developed to the extent allowing for free exploration (except indoor locations that are still in a concept stage).

\section{STRATEGY / METHOD FOR EVALUATING THE SERIOUS GAME}

Evaluating procedural knowledge when failure is potentially life threatening is genuinely problematic. For obvious ethical reasons we cannot expose participant to either live or controlled traffic conditions. A large number of children exposed to the game for a fixed amount of hours, could provide a large enough sample of participants to evaluate if, and to what extent the game helps to increase children road safety based on statistical analysis. However, real life experiment would not only be difficult to conduct but also lengthy and possibly unreliable due to a number of external, uncontrollable and often unknown variables. Therefore, an alternative solution is needed for the purpose of evaluating effectiveness of instruction delivered in "Woodlands". One potential solution is to use a photorealistic Virtual Reality environment designed for evaluating pedestrian behaviour [19], that could serve as a pre, post and delayed test for evaluation of progress, retention and arguably transfer as well. Results of such test should be measured against the overall ratio of successful over total number of attempts, or the highest level of difficulty (controlled by speed and frequency of incoming traffic) at which the learner can still perform safely, rather than the time taken by the participant to successfully cross a road. The time needed to complete each task should decrease with training, but since the game encourage patience over haste this result would likely be a by-product of practice and possibly increased player's confidence. Decreasing the time needed for crossing a road is not an educational goal that "Woodlands" hope to achieve, on the contrary a negative reinforcement could be introduced to a group of participants that would be tested against a group exposed to positive reinforcement and a control group without reinforcement, to evaluate its impact on time taken to make a decision of stepping onto a road, where the hypothesise would be that the mean time needed for completing the task would increase for the group where negative reinforcement was present.

Informal usability testing was performed on a group of children and undergraduate students, primarily to gain insights on functionality of the prototype and stakeholders attitude towards delivering practical road safety education in Virtual Reality.

First stage of testing included a series of micro-tasks (e.g.: start a new game), primarily to evaluate effectiveness of gaze-based interactions [see web resource $34 \& 35$ ]. As a result of these tests a rapid visual feedback (e.g.: change in colour and/or size) was implemented in every feature that utilise gaze-based interactions, to decrease the gulf of execution.

Second stage involved free exploration of the environment or following the basic story line implemented in the beta version being tested. As such, participants were given minimum amount of instruction from the test administrator and qualitative interviews were conducted post-exposure (see [31] for a more detailed description of the testing procedure and findings).

Final testing stage of the prototype was conducted during the Final Year Show where the functional prototype was made available to general public visiting the show, qualitative interviews were performed post-exposure primarily to evaluate stakeholders' attitude towards VR game-based road safety education.

\section{RESULTS}

"Woodlands" never went past the prototype stage, therefore all evaluations performed were informal, mainly focused on usability, and did not involve sufficient sample size to yield any kind of statistically significant results. However, the feedback from both parents and children who play tested the game has been overwhelmingly positive. It seems that the parents are very interested in seeing games developed for educational purposes and they see a merit in addressing road safety procedural knowledge through a Virtual Reality application. However, a more structured and formal study is required to support this notion.

None of the children participating in beta tests [see fig. $5 \& 6$ ] managed to identify the true purpose of "Woodlands", meaning that despite the fact that some components (like 'the lollipop lady') are strongly giving away its nature, they do not perceive it as a road safety exercise. All children participating in informal tests were eager to play the game further and expressed disappointment when the test finished abruptly. This could mean that the design was successful in capturing their imagination. Four out of nine children taking part in the second stage of beta tests asked about the windows and/or doors on the trees. As recommended by functionality testing methodology, instead of being provided with an answer they were asked "how do they think?" instead. All four children answered that there must be people living inside, although three were unable to specify what exactly are they doing in there, one girl answered: "they are having a party", possibly due to an audio source that was located inside that tree.

\section{FURTHER WORK}

Number of components have not been implemented in the last version of the prototype due to time constraints:

1) Broken set of traffic lights (by having to fix the traffic lights the attitude towards using traffic lights should improve).

2) Safe cross code solution and a complete story line.

3) Indoor locations (needed to provide a platform for content expansion).

4) Series of small quests (to increase the number of crossings performed in a game by the player).

Navigating the environment is currently operated either through a gamepad or a mouse and a keyboard. However, mapping player inputs onto a device that is abstract to how human traverse a physical environment is far from ideal. Use of an omni-directional treadmill, would allow players to traverse the 
environment using their legs instead of their fingers. This should increase the transfer of skills to the real world even further and also be more intuitive to the player. Virtual Reality sickness, similarly to other motion-induced illnesses is most likely caused by a sensory conflict (Oman, 1988), therefore more natural form of traversing through the environment should resolve some issues with a mismatch between an artificial in game movement rendered on screen and players proprioception.

Currently implemented gaze-based interactions are far from ideal. As much as it often seems to be the case, there is no guarantee that the player will always keep their gaze in the center of their field of view in the HMD. This might have an impact on accuracy of interactions and can be particularly problematic during spotting cars. Therefore, an eye tracking based solution should be implemented in the system, when a reliable eye tracking hardware can be used with an HMD.

At the time of writing this publication "Woodlands" is still a prototype, and is likely to remain in that stage unless additional funding can be secured for further development. Proof of concept demonstrate basic functionality, including assisted road crossing. However, in order to make it work as intended the game environment and story line has to be vastly expanded to provide players with a purpose for further game play, in consequence leading to an increased number of attempts at crossing the road.

The impact of delayed feedback versus immediate feedback on retention and learning pace in "Woodlands" also requires further investigation.

\section{DISCUSSION}

There is a possibility that by the time VR technology will become prevalent and accessible to most children, self-driving cars will already become a norm. It is our hope that self-driving cars will improve child safety while they are crossing a road, however for the time being it is still a problem that can be solve primarily through means of training. As such one potential solution to accessibility could be addressed by a partnership with Road Safety Authorities who could provide mobile VR stations that could travel between schools offering training without any need of financial investment on the learner side.

Side effects of using HMDs' known as simulator sickness could potentially serve as a safe way of using nausea as a reinforcement mechanism. However, it is unknown how quickly, if ever participants would learn to associate nausea with undesired behaviour, and if it wouldn't be quickly dissociated as it turned out in other basic conditioning cases where drug induced nausea was used to help alcohol addicts.

We hypothesize that VR can utilize environment affordances to facilitate conditioning behaviors and developing habits, as such a more detailed discussion is needed to highlight potential ethical implications of using 'skinner boxes' in games in general, but particularly in VR applications. We have described one potential application that can be used to serve humanity but it doesn't require a lot of imagination to come up with opposite results. If presence truly enables responses as if real and blurs the line between virtual and physical worlds we should carefully consider any potential implications that in might have on human psychology, particularly in Virtual Environments where violence is being rewarded or in cases where sexual content is present.

There also seems to be little data available on the impact of VR exposure on both children and adults. This situation is somewhat concerning as the technology has already entered the consumer market while no longitudinal studies are available to even attempt to predict the consequences it might have on the society and its members in the future.

More research on presence in mediated environments is needed to understand the limitations and capacity of VR technology. We argue that quantitative metrics are urgently needed to evaluate its potential impact not only in education but also in the entertainment domain, as the data available on the impact of violence in video games on child development might not be applicable to VR games.

During the second stage of beta testing, involving flying on a tree leaf from one platform onto another, one of the participants demonstrated symptoms of acrophobia (fear of heights), despite having a fairly strong reaction the participant was keen on continuing and quickly managed to overcome their phobic response (see [31] for more details). As such, we have reasons to believe that game-based approach to exposure therapies might increase the effectiveness of Virtual Reality Exposure Therapies (VRET) for children. However, a more in-depth study is needed to confirm this hypothesis.

\section{CONCLUSION}

The prototype that we have described here serves effectively as a proof of concept and can be used as a foundation for a more structured study into the effectiveness of Virtual Reality serious games, and possible impact of 'presence' on the effectiveness of learning for students using serious games in the context of procedural knowledge acquisition. We hypothesise that Virtual Reality environments might serve as an effective training platform for a variety of psycho-motoric skills that would be either too dangerous or too expensive to practice in a real life (e.g.: fire-fighters or emergency flight procedures for pilots training).

Locke believed that we gain knowledge through experience [32], if our assumptions on what 'presence' is are correct, Virtual Reality might be the very first medium that allows learners to experience knowledge directly as if they would have lived through it. 


\section{Figures}

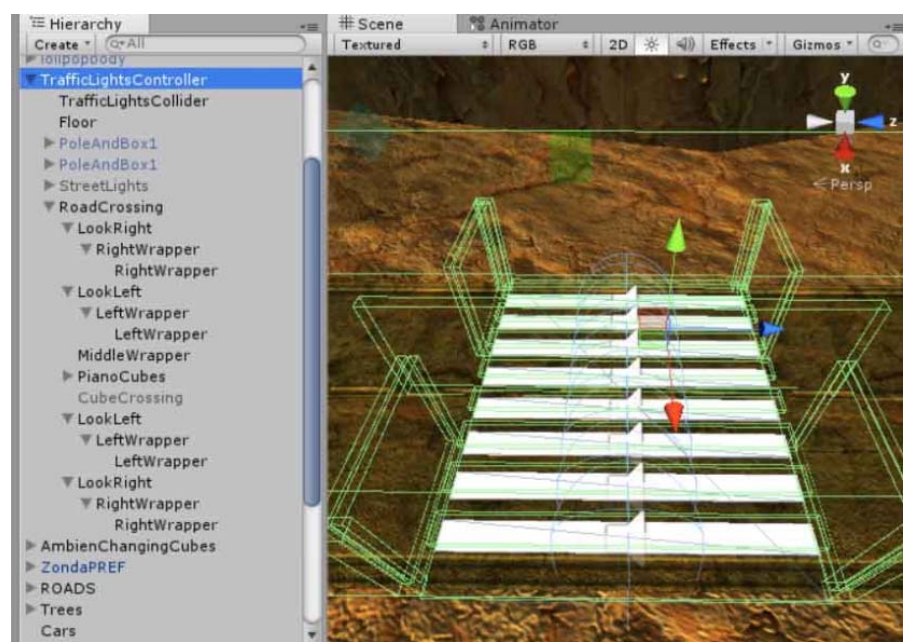

Figure 1: Placement of colliders used for enabling access to the pedestrian crossing

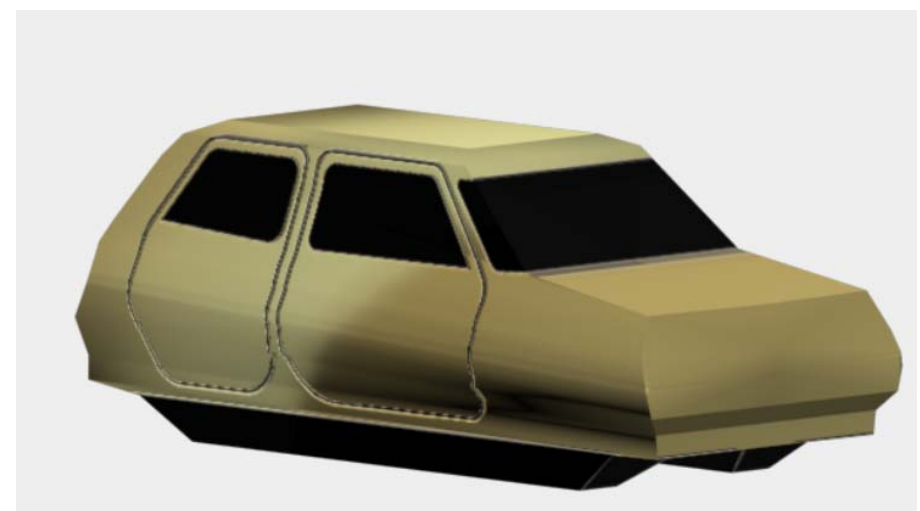

Figure 2: The 3D model of a car with a wood texture applied
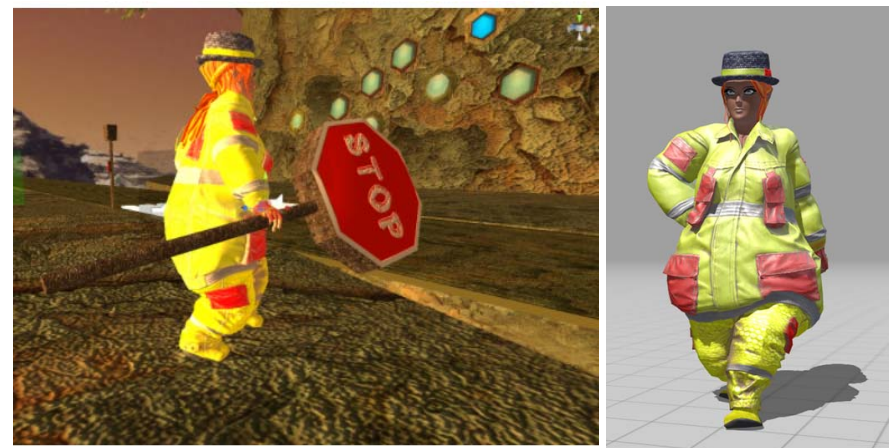

Figure 3 \& 4: The Lollipop Lady NPC
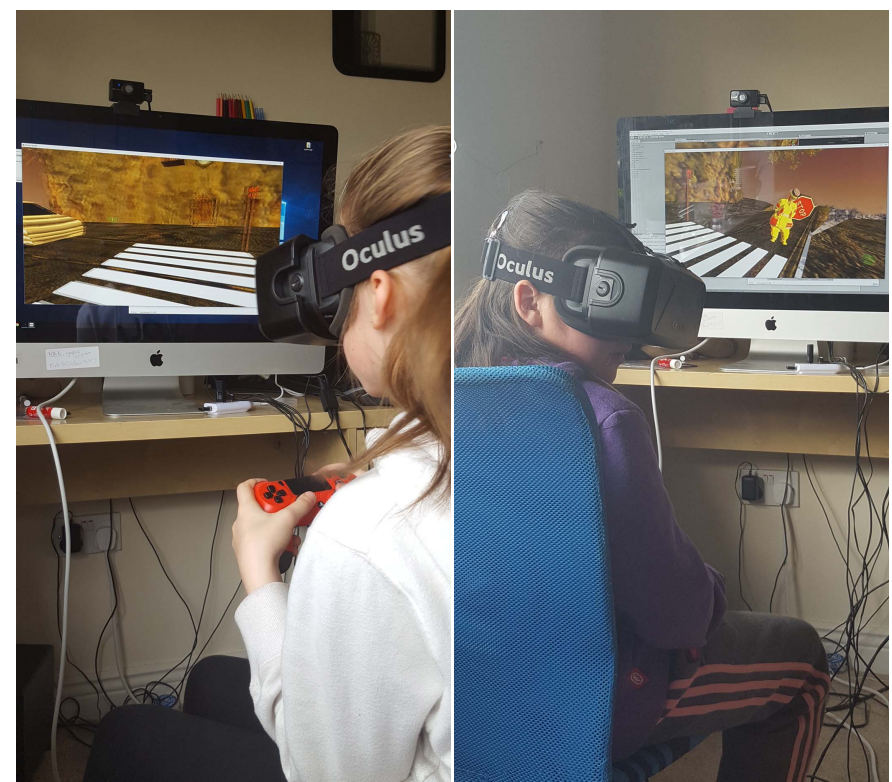

Figure 5 \& 6: Children evaluating "Woodlands" during beta tests

\section{REFERENCES}

[1] RSA, "Road Safety Authority," [Online]. Available: http://www.rsa.ie/RSA/Road-

Safety/Campaigns/Current-road-safety-campaigns/SafeCross-Code/. [Accessed 2018].

[2] T. W. Malone, "Toward a theory of intrinsically motivating instruction," Cognitive science, vol. 5, no. 4, pp. 333-369, 1981.

[3] M. Slater, "Place illusion and plausibility can lead to realistic behaviour in immersive virtual environments," Philosophical Transactions of the Royal Society B: Biological Sciences, vol. 364, no. 1535, pp. 3549-3557, 2009.

[4] N. E. Seymour, A. G. Gallagher, S. A. Roman, M. K. O'brien, V. K. Bansal, D. K. Andersen and R. M. Satava, "Virtual reality training improves operating room performance: results of a randomized, doubleblinded study," Annals of surgery, vol. 236, no. 4, 2002.

[5] D. J. Glisczinski, "Lighting up the mind: Transforming learning through the applied scholarship of cognitive neuroscience," International Journal for the Scholarship of Teaching and Learning, vol. 5, no. 1, 2011.

[6] D. A. Kolb, Experiential learning: Experience as the source of learning and development, FT press, 2014.

[7] M. Raab, "Implicit and Explicit Learning of Decision Making in Sports is Effected by Complexity of Situation," International Journal of Sport Psychology, vol. 34, no. 4, pp. 273-288, 2003.

[8] S. Smith and E. Ericson, "Using immersive game-based virtual reality to teach fire-safety skills to children," Virtual reality, vol. 13, no. 2, pp. 87-99, 2009. 
[9] S. Sharples, S. Cobb, A. Moody and J. R. Wilson, "Virtual reality induced symptoms and effects (VRISE): Comparison of head mounted display (HMD), desktop and projection display systems," Displays, vol. 29, no. 2, pp. 58-69, 2008.

[10] K. M. Kim, Z. Rosenthal, D. Zielinski and R. Brady, "Comparison of desktop, head mounted display, and six wall fully immersive systems using a stressful task," in Virtual Reality Short Papers and Posters (VRW), Costa Mesa, California, 2012.

[11] M. Slater, J. McCarthy and F. Maringelli, "The influence of body movement on subjective presence in virtual environments," Human Factors, vol. 40, no. 3, pp. 469-477, 1998.

[12] R. Garris, R. Ahlers and J. E. Driskell, "Games, motivation, and learning: A research and practice model," Simulation \& gaming , vol. 33, no. 4, pp. 441467, 2002.

[13] M. Prensky, "Digital natives, digital immigrants part 1," On the horizon, vol. 9, no. 5, pp. 1-6, 2001.

[14] R. Van Eck, "Digital game-based learning: It's not just the digital natives who are restless," EDUCAUSE review, vol. 41, no. 2, 2006.

[15] T. Malone, "What makes computer games fun? (Abstract Only)," in Proceedings of the Joint Conference on Easier and More Productive Use of Computer Systems. (Part - II): Human Interface and the User Interface, Ann Arbor, MI, 1981.

[16] R. R. Burton and J. S. Brown, "An investigation of computer coaching for informal learning activities," International Journal of Man-Machine Studies , vol. 11, no. 1, pp. 5-24, 1979.

[17] S. Bull and M. Smith, "A pair of student models to encourage collaboration," in User Modeling, Vienna, 1997.

[18] M. Smith, "What Computing Students Can Learnby Developing Their Own Serious Games," in Serious Games and Edutainment Applications, London, Springer, 2011, pp. 447-480.

[19] K. Szczurowski, ""Crosswalk 2" project - a Virtual Reality simulator for evaluating pedestrian behaviour while making the decission to cross a road," Unpublished, Simulator Research Group (SimRG) at the Insitute of Technology Blanchardstown, Dublin, Ireland, 2015.

[20] M. V. Sanchez-Vives and S. Mel, "From presence to consciousness through virtual reality," Nature Reviews Neuroscience, vol. 6, no. 4, 2005.

[21] K. Szczurowski and M. Smith, "Measuring Presence Hypothethical Quantitative Framework," in Virtual System \& Multimedia (VSMM), 2017 23rd International Conference, Dublin, Ireland, 2017.
[22] W. B. Scoville and B. Milner, "Loss of recent memory after bilateral hippocampal lesions," Journal of neurology, neurosurgery, and psychiatry, vol. 20, no. 1, 1957.

[23] M. G. Packard and B. J. Knowlton, "Learning and memory functions of the basal ganglia," Annual review of neuroscience, vol. 25, no. 1, pp. 563-593, 2002.

[24] C. J. Hooper, M. Luciana, H. M. Conklin and R. S. Yarge, "Adolescents' performance on the Iowa Gambling Task: implications for the development of decision making and ventromedial prefrontal cortex," Developmental psychology, vol. 40, no. 6, 2004.

[25] K. A. Ericsson and N. Charness, "Expert performance: Its structure and acquisition," American psychologist, vol. 49, no. 8, 1994.

[26] Road Safety Authority (RSA), "rsa.ie," [Online]. Available: http://www.rsa.ie/en/RSA/RoadSafety/Campaigns/Current-road-safety-campaigns/SafeCross-Code/Safe-Cross-Code-Song/.

[27] G. Keppel, "Facilitation in short-and long-term retention of paired associates following distributed practice in learning," Journal of Verbal Learning and Verbal Behavior, vol. 3, no. 2, pp. 91-111, 1964.

[28] B. J. Wadsworth, Piaget's theory of cognitive and affective development: Foundations of constructivism (5th ed.), White Plains, NY, England: Longman Publishing, 1996.

[29] L. Maister, M. Slater, M. V. Sanchez-Vives and M. Tsakiris, "Changing bodies changes minds: owning another body affects social cognition," Trends in cognitive sciences, vol. 19, no. 1, pp. 6-12, 2015.

[30] J. Schell, "youtube.com," DICE 2010 conference, 2010. [Online]. Available: https://www.youtube.com/watch?v=nG_PbHVW5cQ.

[31] K. Szczurowski, "Kris Szczurowski online portfolio Testing," 1805 2016. [Online]. Available: http://kris.szczurowski.info/2016/05/18/testing/. [Accessed 2018].

[32] J. Locke, Of the Conduct of the Understanding, Doctoral dissertation: P. Schuurman, 2000.

\section{WEB RESOURCES}

[33] K. Szczurowski, "Beta test \#1", video recording of a beta test with an undergraduate student, youtube.com, 2018 [Online]. Available: https://youtu.be/qE4nYTs4III

[34] K. Szczurowski, "Beta test \#2", video recording of a beta test with an undergraduate student, youtube.com, 2018 [Online]. Available: https://youtu.be/1ipeljAX 